\title{
Adrenergic effects on hypothalamic activity: Alpha and beta agonists and antagonists
}

\author{
HUGH E. CRISWELL \\ Williams College, Williamstown, Massachusetts 01267 \\ and \\ ROBERT A. LEVITT \\ Southern Illinois University, Carbondale, Illinois 62901
}

\begin{abstract}
Dose-response curves were plotted to analyze the effects of adrenergic agonists and antagonists upon neural activity within the hypothalamus. Multiple unit activity (MUA) was recorded following microinjection of the drugs directly into the recording site through a hollow recording electrode. Dose-dependent decreases in MUA occurred following the microinjection of the alpha-adrenergic agonist 1-norepinephrine and also following low, but not high, doses of the alpha-adrenergic antagonist phentolamine. Neither the beta-adrenergic agonist dl-isoproterenol nor the beta-adrenergic antagonist dl-propranolol produced dose-related effects upon MUA at the sites tested. A small nonbiochemically specific decrease in MUA was also found to follow all microinjections into the lateral or ventromedial hypothalamus when any compound was microinjected, including isotonic saline.
\end{abstract}

Adrenergic neural mechanisms within the brain have been the subject of extensive investigation. A deficit in human adrenergic functions has been linked to schizophrenia and the affective disorders (Stein \& Wise, 1971), and animal studies have linked adrenergic neural activity to such diverse phenomena as sleep (Jouvet, 1969), reinforcement (Stein, 1969) and the regulation of food intake (Miller, 1965).

A large body of evidence has accumulated linking adrenergic transmission within the hypothalamus to the regulation of food intake. Stimulation of the lateral hypothalamic area (LHA) either electrically (Delgado \& Anand, 1953) or by the microinjection of norepinephrine (Grossman, 1960) has been shown to elicit food intake. Lesions of the LHA have been shown to disrupt feeding (Anand \& Brobeck, 1951). These data suggest that the LHA may act as a "feeding center" with excitation of the LHA eliciting food intake and a decrease in LHA activity producing a feeding deficit. The excitatory effects of the LHA upon food intake have been hypothesized to be under inhibitory control of a "satiety center" located in the ventromedial nucleus of the hypothalamus (VMN). Electrical stimulation of the VMN has been shown to suppress food intake (Wyrwycka \& Dobrezcka, 1960) and bilateral lesions of the VMN produce overeating and obesity (Anand \& Brobeck, 1951).

Recent studies have cast doubt on two aspects of the theory that feeding is controlled by an. LHA adrenergic "feeding center" under the inhibitory control of a VMN "satiation center." Lesions which destroy the fiber tracts which pass between the VMN and the LHA (the ventral

This research was supported by grants from the National Institute of Mental Health of the United States (MH 14381) and the Southern Illinois University Office of Research and Projects. noradrenergic bundle), but which spare the VMN, produce hyperphagia (Ahlskog \& Hoebel, 1973; Gold, 1973), while lesions which spare the fiber tracts while destroying the VMN do not produce hyperphagia (Gold, 1973). Thus, the VMN may not contain an inhibitory mechanism for the control of food intake as was earlier thought, but may simply be near to the ventral noradrenergic bundle.

The ventral noradrenergic bundle is known to supply adrenergic innervation to the LHA (Ungerstedt, 1971), which suggests that norepinephrine may inhibit rather than excite LHA neural activity. Just such a theory has been presented by Margules $(1969,1970$ a) who found that when norepinephrine was microinjected into the LHA at night (when rats normally feed) a decrease in food intake resulted. The paradoxical effect of increased food intake following adrenergic stimulation of the LHA during the day and a decrease in food intake following adrenergic stimulation of the LHA at night was posited to result from an interaction of the adrenergic stimulation with the endogenous levels of norepinephrine in the LHA. Adrenergic stimulation at night, when systemic levels of norepinephrine are highest, was hypothesized to produce an additive effect; while adrenergic stimulation during the day, when endogenous norepinephrine levels were low, may simply have increased the effectiveness of the reuptake mechanism and have actually reduced the overall level of adrenergic stimulation (Margules, Lewis, Dragovich, \& Margules, 1972). The theory that high levels of adrenergic stimulation of the LHA result in a decrease in feeding behavior is consistent with the known anorexic effects of amphetamine. Amphetamine produces anorexia following either systemic administration or direct microinjection into the LHA (Booth, 1968), 
presumably as a result of the release of large amounts of norepinephrine from adrenergic presynaptic terminals. In agreement with the interaction theory of the Margules group (1972), small amounts of amphetamine sometimes produce an increase rather than a decrease in food intake (Glick \& Muller, 1971).

An inhibitory effect of norepinephrine upon LHA neural activity would be consistent with the known inhibitory effect of norepinephrine upon the majority of neurons in other brain structures (Kent, 1973; Moss, Urban, \& Cross, 1972; Segal \& Bloom, 1974). The effects of norepinephrine on food intake appear to be the result of an alpha-adrenergic mechanism, as the alpha-adrenergic blocking agent phentolamine produces hyperphagia when microinjected into the LHA during the dark, a response opposite to that of norepinephrine. Neither a beta-adrenergic agonist (isoproterenol), nor a beta-adrenergic blocking agent (propranolol) produced this effect (Margules, 1970a, 1970b).

To attempt to obtain a clearer understanding of the role of adrenergic agents in the regulation of feeding, the present study examines the effects of alpha- and beta-adrenergic agonists and antagonists upon neural activity within the LHA and VMN.

\section{METHOD}

\section{Subjects}

Seventy-two adult male Long-Evans strain rats, weighing between 450 and $550 \mathrm{~g}$ were maintained in individual cages of a 12-h-light/12-h-dark cycle with food and water available ad lib until the time of surgery. All experiments were conducted during the day period.

\section{Surgery}

Each rat was deeply anesthetized with sodium pentobarbital (Nembutal $70 \mathrm{mg} / \mathrm{kg}$ ) and placed in a stereotaxic instrument. The scalp was incised and $1-\mathrm{mm}$ burr holes were placed bilaterally $2.0 \mathrm{~mm}$ posterior to bregma and $.7 \mathrm{~mm}$ lateral to the midline (VMN placements). Two additional burr holes were placed bilaterally, $3.0 \mathrm{~mm}$ posterior to bregma and $1.8 \mathrm{~mm}$ lateral to the midline (LHA placements).

An array of chemitrodes was then lowered through the burr holes to a depth at which their tips rested bilaterally in the perifornical LHA and VMN sites. A stainless steel jeweler's screw was placed $4 \mathrm{~mm}$ anterior to bregma on the midline and served as an indifferent electrode.

\section{Electrodes and Recording Equipment}

Microinjections and electrical recording were accomplished by a method previously described by Buerger, Levitt, and Irwin

Table 1

The Number of Neuronal Populations Sampled for Each Drug Expressed as the Number of Samples from the LHA Over the Number from the VMN (LHA/VMN)*

\begin{tabular}{ccccc}
\hline $\begin{array}{c}\text { Dose } \\
\text { (Micrograms) }\end{array}$ & $\begin{array}{c}\text { Norepine- } \\
\text { phrine }\end{array}$ & $\begin{array}{c}\text { Phento- } \\
\text { lamine }\end{array}$ & $\begin{array}{c}\text { Isopro- } \\
\text { terenol }\end{array}$ & $\begin{array}{c}\text { Pro- } \\
\text { panolol }\end{array}$ \\
\hline 10 & $6 / 0$ & $7 / 7$ & $7 / 7$ & $4 / 5$ \\
1 & $6 / 0$ & $5 / 3$ & $5 / 4$ & $4 / 2$ \\
.1 & $5 / 5$ & $5 / 5$ & $5 / 5$ & $5 / 0$ \\
\hline
\end{tabular}

*Saline control $=5 / 5$
(1973). An array of four chemitrodes, fabricated from four 30-ga. cannulae, insulated except at their tips, was lowered into the selected sites. Electrical activity from the tip of each electrode was fed into a Grass P17 preamplifier with an amplification factor of 100 , a lower half-amplitude frequency of $300 \mathrm{~Hz}$, and an upper half-amplitude frequency of $3,000 \mathrm{~Hz}$. This system of filtering eliminated EEG activity, revealing the superimposed spike activity from the population of cells near the tip of the electrode (Irwin \& Criswell, 1974). The filtered spike activity was then further amplified and integrated by a Grass 7P3 amplifier-integrator, and the integrated record of multiple unit activity (MUA) was displayed on a Grass Model 7 polygraph. To facilitate the comparison of results from several subjects, an on-line calibration signal was superimposed on the electrical activity from each site (Irwin \& Criswell, 1974).

\section{Procedure}

Following surgical preparation, each subject was transferred from the operating room to an electrically shielded recording room, the chemitrodes were attached by alligator clips to the preamplifiers and recording was begun. Recording 'vas continued until a steady baseline of neural activity was reached. An on-line calibration signal of 2 microV sec was passed through each electrode, and, following the calibration, a 15-min baseline period was recoraed at a paper speed of $25 \mathrm{~mm} / \mathrm{min}$.

Following the 15-min baseline period, a microinjection was made through the hollow chemitrode into one of the four recording sites. Recording was continued for $30 \mathrm{~min}$ following the microinjection. At this time, if the subject remained deeply anesthetized (did not respond to a strong foot pinch), a microinjection was made in to one of the four sites and recording was continued for an additional $30 \mathrm{~min}$. In some cases, after MUA had recovered ( 1 to $2 \mathrm{~h}$ ), a drug injection was made into one of the two sites on the contralateral side of the brain and a second set of data was obtained.

\section{Drugs}

The adrenergic drugs employed in the present study included an adlpha-adrenergic agonist (L-norepinephrine $\mathrm{HCL}$, L-Arterenol; Sigma), and alpha-adrenergic antagonist (phentolamine, Regitine Mesylate; CIBA), a beta-adrenergic agonist (DL-isoproterenol HCL, Sigma), and a beta-adrenergic antagonist (DL-propranolol HCL, Sigma). Doses of each drug we re .1 microgram, 1.0 microgram, and 10.0 micrograms dissolved in .5 microliters of isotonic saline. A group of 10 subjects received microinjections of the saline injection medium alone to control for the physical effects of the microinjection process. The drugs used, the doses employed, and the number of populations sampled at each site are shown in Table 1.

\section{Histology}

Immediately after the recording session, subjects were perfused with normal saline followed by fixation in $10 \%$ formal saline. Following fixation, 40-micron frozen sections were stained with cresyl violet and the position of the electrodes was verified. Only subjects with electrodes verified to be in the LHA or the VMN were used in the data analy sis.

\section{RESULTS}

Each animal had an array of four microelectrodes. In many cases, all four electrodes were successfully recorded from. However, the effects recorded from noninjected sites were invariably in the same direction as when recordings were made from the site of injection, except that changes in the MUA at noninjected sites tended to be smaller. Furthermore, similar changes were 


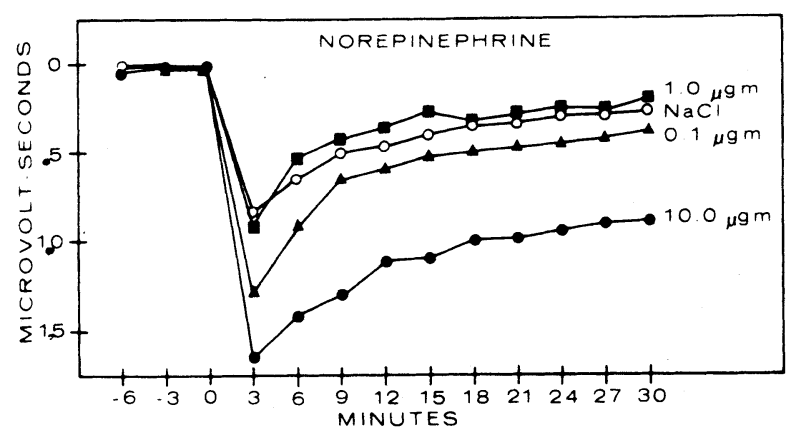

Figure 1. Multiple unit activity changes, produced by norepinephrine microinjections into the LHA or VMN (the drug was injected at Time 0 ).

produced in noninjected sites ipsilateral and contralateral to the site of injection. Since, additionally, very similar changes were produced by VMN and LHA injections at the sites of injection, the data in Figures 14 have been collapsed over and include both LHA and VMN injection sites, and we are not reporting the data from the noninjected sites.

Dose-response plots of the effects on MUA from the site of microinjection of an alpha-adrenergic agonist or antagonist injected directly into the hypothalamus are shown in Figures 1 and 2. There was a significant change in the effect of the microinjection of 1-norepinephrine as a function of time, when MUA levels immediately following the microinjection were compared with those $15 \mathrm{~min}$ and $30 \mathrm{~min}$ later $(\mathrm{F}=79.0, \mathrm{df}=2 / 58, \mathrm{p}<.01)$. It can be seen that the decrease in MUA which followed the microinjection recovered with time. There was also a differential effect among the doses employed $(F=3.39$, $\mathrm{df}=3 / 29, \mathrm{p}<.05)$. Tukey tests indicated that only the 10-microgram dose produced a change in MUA significantly different than the saline control $(p<.05)$. There was no interaction between the doses employed and time on the MUA changes following the microinjection of 1 -norepinephrine.

There was also a significant effect of the microinjection of the alpha-adrenergic antagonist phentolamine upon MUA as a function of time $(\mathrm{F}=65.85, \mathrm{df}=2 / 78, \mathrm{p}<.01)$, and there was a

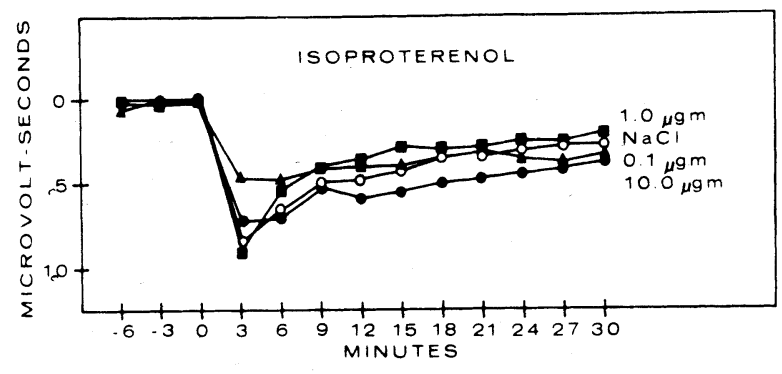

Figure 3. Multiple unit activity changes produced by isoproterenol microinjections into the LHA or VMN (the drug was injected at Time 0 ).

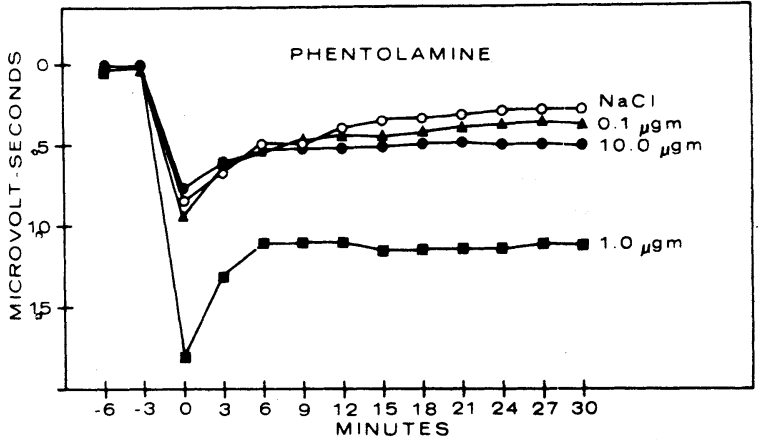

Figure 2. Multiple unit activity changes, produced by phentolamine microinjections into the LHA or VMN (the drug was injected at Time 0 ).

differential effect upon MUA of the various doses employed $(F=7.06, \mathrm{df}=3 / 39, \mathrm{p}<.01)$. Tukey tests indicated that only the 1.0 -microgram dose produced a decrease in MUA which was significantly different than the saline control injection $(\mathrm{p}<.05)$. There was no interaction between dose and time for the effect of phentolamine upon MUA. Note that both the alpha-adrenergic agonist and the antagonist produced decreases in MUA.

Dose-response plots of the effects of microinjecting a beta-adrenergic agonist (dl-isoproterenol) and a beta-adrenergic antagonist (dl-propranolol) directly into the hypothalamus upon MUA from the site of the microinjection are shown in Figures 3 and 4. There was a significant decrease in MUA, which recovered over time, following the microinjection of dl-isoproterenol $(\mathrm{F}=36.25, \quad \mathrm{df}=2 / 54, \mathrm{p}<.01) \quad$ or dl-propranolol $(\mathrm{F}=24.45, \mathrm{df}=2 / 52, \mathrm{p}<.01)$, but the decrease in MUA following the microinjection of these two drugs did not differ from that following the microinjection of the isotonic saline. There was also no interaction between drug dose and time for either the dl-isoproterenol or the dl-propranolol.

\section{DISCUSSION}

Decreases in MUA followed the microinjection of isotonic saline or any of the doses of each of the four drugs which were investigated. However, only the alpha-adrenergic drugs produced effects significantly different than the saline control.

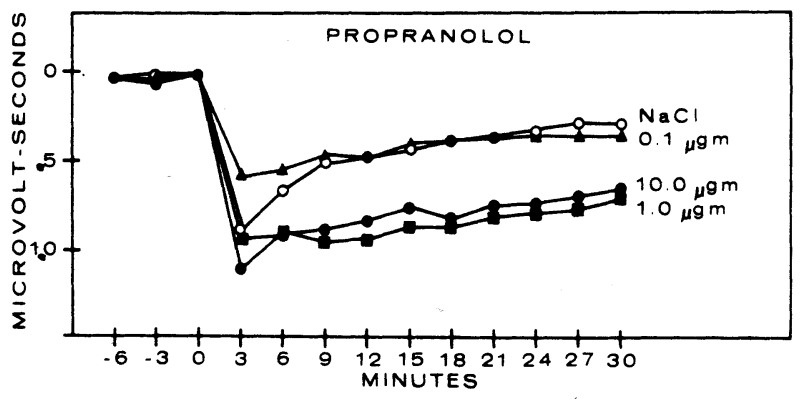

Figure 4. Multiple unit activity changes produced by propranolol microinjections into the LHA or VMN (the drug was injected at Time 0). 
Norepinephrine at a dose level of 10 micrograms produced a greater decrease in MUA than did the saline control. This is in agreement with previous studies of the effects of norepinephrine upon neural activity. These studies have almost universally found norepinephrine to be an inhibitor of the majority of neurons tested (Kent, 1973; Moss, Urban, \& Cross, 1972; Segal \& Bloom, 1974).

The decrease in MUA following the 1.0-microgram dose of phentolamine is somewhat surprising in light of its blocking effect at the alpha-adrenergic synapse. Neither the .1 -microgram dose nor the 10.0-microgram dose of phentolamine produced a significant effect. This suggests that the dose-response curve for the action of phentolamine may not be monotonic and that small doses in the neighborhood of the 1.0-microgram dose examined in the present study may produce an activation of the alpha-adrenergic synapse similar to that following stimulation by an alpha-adrenergic agonist, while higher doses are required to produce the alpha-adrenergic blockade which is commonly seen following the administration of phentolamine.

The lack of effect of the beta-adrenergic agonist and antagonist suggests that the majority of hypothalamic neurons which are responsive to adrenergic stimulation are part of an alpha-adrenergic system.

A direct comparison of the effects of the four drugs on the two different hypothalamic areas (LHA vs. VMN) was not an integral part of the present experiment and the number of observations from each site varied as a function of both drug type and dose, as can be seen in Table 1 . However, the raw data did not show any indication that the LHA and VMN showed differential modes of response to the drugs. All microinjections at each site produced an initial decrease in MUA with the exception of two samples of neurons in the LHA which did not respond to the microinjection. By the end of the 30-min recording period, only 2 of the 62 sites in the LHA had returned to a level of neural activity above the preinjection baseline, while 1 of the 46 sites in the VMN reached a level above the original baseline. The similarity of effects of the drugs between the two structures does not lend support to theories suggesting that adrenergic stimulation has opposite effects on the LHA and VMN.

\section{REFERENCES}

Ahlskog, J. E., \& Hoebel, B. G. Overeating and obesity from damage to a noradrenergic system in the brain. Science, 1973, $182,166-168$.

Anand, B. K., \& Brobeck, J. R. Hypothalamic control of food intake in rats and cats. Yale Journal of Biology and Medicine, $1951,24,123-138$.

Booth, D. A. Amphetamine anorexia by direct action on the adrenergic feeding system of rat hypothalamus. Nature, 1968, 217, 869-870.
Buerger, P. B., Levitt, R. A., \& Irwin, D. A. Chemical stimulation of the brain: Relationship between neural activity and water ingestion in the rat. Journal of Comparative and $\mathrm{Phy}$ siological Psy chology, 1973, 82, 278-285.

Delgado, J. W., \& Anand, B. K. Increase of food intake induced by electrical stimulation of the lateral hypothalamus. American Journal of Physiology, 1953, 172, 162-168.

Glick, S. D., \& Muller, R. U. Paradoxical effects of low doses of d-amphetamine in rats. Psychopharmacologia, 1971, 22, 369-402.

Gold, R. M. Hypothalamic obesity: The myth of the ventromedial nucleus. Science, $1973,182,488-490$.

Grossman, S. P. Eating or drinking elicited by direct adrenergic or cholinergic stimulation of the hypothalamus. Science, 1960, $132301-302$.

Irwin, D. A., \& Criswell, H. W, A system for accurate on-line calibration of multiple unit potential recording. Physiology and Behavior, 1974, 12, 703-705.

Jouvet, M. Biogenic amines and the states of sleep. Science, $1969,163,32-41$.

Kent, E. W. Reticular Units: Relations between responses to sensory stimulation and responses to neurohumors. Behavioral Biology, 1973, 8, 227-228.

Margules, D. L. Noradrenergic synapses for the suppression of feeding behavior. Life Sciences, 1969, 8, 693-704

Margules, D. L. Alpha-adrenergic receptors in hypothalamus for the suppression of feeding behavior by satiety. Journal of Comparative and Physiological Psychology, 1970, 73, 1-12. (a)

Margules, D. L. Beta-adrenergic receptors in the hypothalamus for learned and unlearned taste aversions. Journal of Comparative and Physiological Psychology, 1970, 73, 13-21. (b)

Margules, D. L., Lewis, M. J., Dragovich, J. A., \& Margules, A. S. Hypothalamic norepinephrine: Circadian rhythms and the control of feeding behavior. Science, 1972, 178, 640-643.

Miller, N. E. Chemical coding of behavior in the brain. Science, $1965,148,328-338$.

Moss, R., Urban, I., \& Cross, B. A. Microelectrophoresis of cholinergic and aminergic drugs on paraventricular neurons. American Journal of Physiology, 1972, 223, 310-318.

Segal, M., \& Bloom, F. E. The action of norepinephrine in the rat hippocampus. I. Ionotophoretic studies. Brain Research, $1974,72,79-97$.

Stein, L. Chemistry of purposive behavior. In J. T. Tapp (Ed.), Reinforcement and behavior. New York: Academic Press, 1969.

Stein, L., \& Wise, C. D. Possible etiology of schizophrenia: Progressive damage to the noradrenergic reward system by 6-hydroxydopamine. Science, 1971, 171, 1032-1036.

Ungerstedt, U. Stereotaxic mapping of monoamine pathways in the rat brain. Acta Physiologica Scandinavica, 1971, 367, $1-48$.

Wyrwycka, W., \& Dobrezcka, C. Relationship between feeding and satiation centers of the hypothalamus. Science, 1960, $132,805-806$.

(Received for publication February 22, 1975.) 